\title{
LAS REDES DE EMPRESAS EN LA INDUSTRIA ELECTRÓNICA DE LA COMUNIDAD DE MADRID
}

\author{
POR \\ RUTH RAMA DELLEPIANE Y ANA MELERO GUILLÓ
}

\section{Introducción}

Objetivos. La finalidad de este trabajo es constatar la existencia de redes de establecimientos en la industria electrónica de la Comunidad de Madrid y determinar su naturaleza*. Partimos de la hipótesis de que la Comunidad de Madrid es una región compuesta de más de un sistema productivo local en lo que respecta al sector de la electrónica (Storper y Harrison, 1991): En la región coexistirían establecimientos electrónicos ligados por la cooperación y localizados en los distritos metropolitanos, y establecimientos con interacciones supra-regionales, incluso nacionales e internacionales, que se encontrarían en los municipios periféricos.

Para desarrollar esta hipótesis, utilizaremos datos estadísticos, estudios publicados, materiales proporcionados por firmas y organizaciones profesionales así como resultados preliminares de una encuesta

\footnotetext{
* Este trabajo fue realizado en el marco de los proyectos SEC97-1373 de la CICYT y 06/0092/1997 de la CAM. Las autoras agradecen a Ascensión Calatrava y a dos evaluadores anónimos por los comentarios realizados a una primera versión del artículo, así como a Manuel Duque por la ayuda proporcionada en la búsqueda y sistematización de la información.
}

Ruth Rama Dellepiane y Ana Melero Guilló: Instituto de Economía y Geografía (CSIC).

Estudios Geográfico

Tomo LXI, 2000, n. ${ }^{\circ} 240$, julio-septiembre 
y de entrevistas realizadas en diferentes establecimientos electrónicos de la Comunidad madrileña ${ }^{1}$. Dada la dimensión regional del estudio, el establecimiento (y no la empresa) constituye, en nuestra opinión, la unidad analítica más representativa de los fenómenos de redes ${ }^{2}$.

La noción de «red» utilizada en este estudio. En la bibliografía sobre este tema, la definición de los fenómenos de redes es muy variada. Es útil, por tanto, identificar desde el principio los fenómenos que incluimos en la noción de red, tal como es utilizada en este estudio. Consideramos los siguientes fenómenos:

Acuerdos de cooperación explícitos entre establecimientos que no pertenecen al mismo grupo, que se ligan en alianzas estratégicas para hacer juntos marketing, I+D, exportar sus productos, desarrollar proyectos en común.

Partenariados comprometiendo la subcontratación.

Relaciones de establecimientos electrónicos con sus clientes y proveedores.

Hay que hacer notar una gran diferencia entre el concepto adoptado este estudio, que comprende relaciones de mercado (con proveedores y clientes), y la noción de red utilizada por otros autores como Cooke y Morgan (1993), que sólo incluye aquellas relaciones que no se dan ni en el mercado ni en las jerarquías ${ }^{3}$. Hemos preferido retener una noción más amplia - y ciertamente menos «pura» desde el punto de vista conceptual- en la medida que ella nos permite delimitar mejor las interrelaciones de establecimientos a escala local.

\footnotetext{
${ }^{1}$ Encuestas de las industrias electrónicas de las Comunidades Autónomas de Madrid, Cataluña y País Vasco, en curso en el IEG-CSIC. La muestra sobre la que se basa este trabajo comprende 50 establecimientos, lo que supone alrededor del $23 \%$ de los establecimientos del sector en la CAM. Sobre la metodología utilizada para seleccionar los establecimientos encuestados, ver Calatrava y Melero, 1999. Compararemos estos resultados de 1999 con los de la encuesta realizada por una de las autoras y el Profesor Suárez-Villa en 1993 (Suárez-Villa y Rama, 1996). Esta última incluye 64 establecimientos electrónicos regionales (34\% de la población en 1993). Habida cuenta de que la encuesta de 1999 está aún en curso, la información presentada en este trabajo es de naturaleza cualitativa.

${ }^{2}$ Cuando la sede de la casa matriz esté en Madrid, la empresa agrupará en general, la información de las fábricas que controla en toda España; por tanto, el análisis de datos a nivel de la empresa correría el peligro de diluir manifestaciones de las interrelaciones de los establecimientos madrileños de la firma y relaciones que estos mantengan con su entorno regional.

${ }^{3}$ Sin embargo, estos autores analizan las relaciones a largo plazo entre usuarios y productores, como una forma de adquisición del learning-by-using (Cooke y Morgan, 1993, p. 549).
} 
Por otro lado, no incluiremos en el concepto de red ni las empresas conjuntas (joint-ventures), ni las licencias de explotación, ni los acuerdos con el sector público (sí incluidos en la definición de Freeman, 1991). Tampoco incluiremos las relaciones externas de naturaleza informal de los establecimientos, que tienen más que ver con las relaciones entre individuos (por ejemplo, las relaciones profesionales entre ingenieros y personal de $\mathrm{I}+\mathrm{D}$ de distintos establecimientos). No consideraremos, pues, ni las situaciones consolidadas asentadas sobre la inversión en el capital de las empresas o sobre los contratos de utilización de la tecnología, ni tampoco las situaciones muy desdibujadas puramente imbricadas en relaciones informales, localizadas o del medio, que a veces son definidas como redes en un sentido más amplio (ver, por ejemplo, Freeman, 1991; Héraud y Nanopoulos, 1994) ${ }^{4}$.

Plan de trabajo. Presentaremos primero la región de Madrid y su industria electrónica. Después, definiremos la naturaleza de los establecimientos electrónicos de la región y su base geográfica. A continuación, analizaremos los fenómenos de redes, cuya lógica de funcionamiento trataremos de explicar. En las conclusiones, intentaremos reflexionar sobre la contribución del estudio de caso de Madrid al conocimiento de los fenómenos de redes.

\section{Presentación de la región estudiada y de su industria electrónica}

La Comunidad Autónoma de Madrid. Con una población de 5 millones de habitantes (12,6\% de la población española) y el 15,9\% del PIB nacional ${ }^{5}$, la Comunidad de Madrid (CAM) ha sido, durante los dos últimos decenios, una de las regiones más dinámicas de la UE. Durante los años 80, principalmente, la tasa de crecimiento de esta región ha estado claramente por encima de la de las regiones de la Europa de los Doce, únicamente superada por las de Île de France y el Lazio italiano (Iranzo et al., 1993).

\footnotetext{
${ }^{4}$ Evidentemente, el tipo de red en el sentido restringido que estudiamos en este trabajo está muy a menudo asociado con ese otro tipo de red informal en el que la proximidad geográfica facilita los intercambios de información y los «spillovers» de conocimientos técnicos.

${ }^{5}$ Datos de 1996 (Consejo Económico y Social de la CAM, 1996) y Boletín de Coyuntura de la CAM, 1997).
} 
Hacia 1985, se puede situar un giro hacia un nuevo modelo productivo regional en el cual es primordial el desarrollo de las nuevas tecnologías. Se trata de una etapa de modernización industrial caracterizada por el desarrollo muy dinámico de las actividades ligadas a los nuevos paradigmas tecnológicos, sobre todo los de la electrónica y las telecomunicaciones. La vocación por la alta tecnología demostrada por la CAM es evidente si se analizan sus datos de gastos en $\mathrm{I}+\mathrm{D}$, que representan más de un tercio del total nacional en 1997 (INE, 1997). Los gastos en $\mathrm{I}+\mathrm{D}$ respecto al PIB de Madrid son del orden del 1,5\%, claramente por encima de la media nacional $(0,87 \%)$ e incluso de la media de la UE (1,34\%). Los indicadores de producción científica, de personal ocupado en actividades de $\mathrm{I}+\mathrm{D}$, de productividad de recursos destinados a la innovación, etc., son igualmente elocuentes en cuanto a la supremacía de Madrid en el territorio español (Buesa y Molero, 1998).

Nacimiento y evolución de la industria electrónica regional. El inicio de la industria electrónica de la CAM data de los años veinte, con la creación de Telefónica, la sociedad semi-pública que tuvo el monopolio de los servicios de telecomunicaciones en España hasta los años 90. La consolidación de esta industria, representada principalmente por la fabricación de equipos para las telecomunicaciones, tuvo lugar en los años 60 con el desarrollo de sectores conexos como la electromedicina, la electrónica de defensa y los componentes electrónicos. La concentración de esta industria en Madrid se deriva de la presencia, en la capital, de su principal cliente, la Administración, por la intermediación de Telefónica y de los Ministerios de Sanidad y de Defensa.

El capital extranjero representa al principio un papel bastante importante en la industria electrónica de la CAM. Una compañía americana, International Telephone and Telegraph (ITT), y su filial española, Standard Eléctrica S. A., se lanzan a la fabricación de equipo de telecomunicaciones en una situación muy favorable, ya que ambas controlaban un mercado monopolístico. Esta situación era diferente de las de la mayoría de los países europeos en aquellos momentos, en los cuales, en general, el Estado controlaba esta industria a través de empresas públicas o de participaciones en el capital de compañías privadas (Suárez Villa y Rama, 1996). Sin embargo, en 1946, el Estado adquiere el 47\% del capital de la filial española de ATT, la cual ha continuado representando un papel muy importante en esta industria en los años cincuenta y sesenta. A comienzo de los años setenta, el Estado creó INTELSA (Industrias de 
Telecomunicación S. A.), una empresa pública que opera bajo licencia de Ericsson y de otras multinacionales. A partir de ese momento, el Estado español comienza a tener un importante papel en el ámbito de la fabricación de equipos para telecomunicaciones. El desarrollo de INTELSA se ha visto estimulado por un mercado muy protegido para el equipamiento y por el monopolio de Telefónica sobre los servicios y las telecomunicaciones. La presencia de esta empresa así como la de varias firmas importantes de comunicaciones y electrónica de defensa, como Telettra y Marconi, estimuló la proliferación de un grupo de pequeñas y medianas empresas (PYMEs) ligadas a las grandes firmas (Suárez-Villa y Rama, 1996).

Los años ochenta y noventa están marcados por fuertes crisis, derivadas no sólo de la repercusión de fluctuaciones internacionales del sector sino también de la concurrencia de productos importados tras la adhesión de España a la CEE en 1986. Sin embargo, también se abrieron nuevas posibilidades para la industria electrónica de Madrid, principalmente las originadas por el crecimiento muy rápido de la demanda de servicios de telecomunicaciones en España y por la expansión internacional de Telefónica. De pronto, las empresas de equipo de telecomunicaciones de Madrid, recobraron su posición de abastecedoras de una de las empresas de servicios de telecomunicaciones más dinámicas y más rentables de Europa.

Aunque con bastante retraso con relación al resto de la UE, la liberación de los servicios de telecomunicaciones en España tuvo, desde su anuncio, un efecto sustancial sobre las estrategias de Telefónica, que se vio obligada a diversificar sus compras de equipo, guiadas a menudo, hasta entonces, por criterios más políticos que económicos. En general, sus proveedores «oficiales» habían sido grandes firmas nacionales de equipos de telecomunicación, en cuyo capital participaba Telefónica, o bien eran multinacionales con filiales en España. En previsión de un mercado más competitivo para los servicios, Telefónica se vio obligada a ajustar sus costes y a racionalizar sus compras de bienes de equipo. Por esa razón, en los últimos años los proveedores locales ya no han sido elegidos sistemáticamente. En 1994-95, Telefónica toma contacto, por primera vez, con «nuevos» abastecedores, como Nokia y Nortel, y comienza a abandonar la fabricación de equipos de telecomunicación para especializarse en los servicios. Por fin, los abonados pueden, por primera vez, comprar directamente a los fabricantes sus aparatos de te- 
léfono y de fax. Eso ha supuesto un gran giro en las condiciones de mercado de ese tipo de equipamiento pues, anteriormente, el usuario no podía adquirirlos más que a través de Telefónica, a menudo a un precio exorbitante. A comienzos de los años noventa, se pasa, por tanto, de un mercado casi monopsónico controlado por Telefónica, a un mercado más concurrencial en el que participan numerosos actores ${ }^{6}$ : los rivales de Telefónica en el mercado de servicios, los operadores de nuevos servicios, como la telefonía móvil, y los abonados. Simultáneamente, se produce una diversificación de las empresas que ofrecen ese tipo de productos en el mercado español ocasionada por la apertura de fronteras y por la desaparición de las listas de proveedores «oficiales» de Telefónica. Hacia mediados de los años noventa, la compañía se lanza a una nueva fase de licitaciones y concursos para adquisición de equipo (Rama, 1998).

La liberalización tiene una influencia muy positiva sobre la caída de los precios de los servicios de telecomunicaciones, que habían estado entre los más elevados de Europa, dando una gran expansión a la demanda. El número de líneas por 100 habitantes ha aumentado de 32,6 en 1990 a 42,76 en 1996 (de 42,1 a 52,3 en Madrid) ${ }^{7}$ (Delgado Rodríguez, 1998). Esos ratios están aún lejos del nivel de saturación, circunstancia que permite prever todavía un período de crecimiento $^{8}$. A su vez, aunque con un efecto retardado y en un entorno de concurrencia redoblada, la liberalización ha abierto nuevos mercados a las empresas de equipamiento de telecomunicaciones de Madrid.

Los principales abastecedores de equipamiento de telecomunicaciones del mercado español son, por orden de importancia: Ericsson, Alcatel, Lucent, Motorola, Amper, Siemens, Nokia y Nortel (1997). En el transcurso del proceso de liberalización, y a pesar del indudable peso de las filiales de las multinacionales y de las grandes empresas nacionales, el Estado ha tratado de preservar «nichos» de mercado para las PYMEs de la industria de equipamientos, que está localizada sobre todo en la CAM. El segundo operador de telefonía móvil que entra en el mercado

\footnotetext{
${ }^{6}$ No obstante, Telefónica sigue siendo el principal comprador, con el $85 \%$ de las compras (Hidalgo Nuchera, 1997).

${ }^{7}$ Según las Comunidades Europeas, España es, en términos de tamaño, el $5^{\circ}$ mercado europeo de servicios de telecomunicación después de Francia, Italia, Alemania y Reino Unido.

${ }^{8} 62$ líneas por cada 100 habitantes en Dinamarca y una relación de 56/100 en Francia, por ejemplo.
} 
español, Airtel ${ }^{9}$, (participado por la británica Vodafone y por su rival British Telecom), tiene contratos directos con 50 PYMEs nacionales, especialmente fabricantes de tarjetas, software y antenas. Además, las multinacionales que le abastecen del equipo de telecomunicaciones están obligadas a subcontratar con PYMEs al menos el $20 \%$ de los trabajos a realizar, como condición fijada por el gobierno español en el momento de la licitación (Rama, 1998).

Durante los años noventa, hay que señalar también las nuevas posibilidades exportadoras que se han abierto a los fabricantes de equipos de telecomunicaciones debidas a la expansión internacional de Telefónica ${ }^{10}$, que se ha introducido, en esos años, en la privatización y la modernización de los sistemas de telecomunicaciones de varios países de Latinoamérica, entre ellos y últimamente Brasil (Rama, 1998) ${ }^{11}$. En 1995, cerca del $50 \%$ de la producción del subsector de equipo de telecomunicaciones de la CAM se exportaba, lo que da una idea de la competitividad de esta industria (Hidalgo Nuchera, 1997). La apertura de Telefónica al mercado internacional, así como la puesta al día tecnológica (digitalización de las líneas) en España, han atraído numerosas inversiones extranjeras en el sector de equipamiento. Ciertas multinacionales han instalado, incluso, divisiones especializadas en Madrid, con vistas a la exportación o a la inversión directa en el mercado latinoamericano y en el marco de los encargos a Telefónica. Sin embargo, no solamente las grandes empresas se han beneficiado de ese potencial abierto por la expansión del antiguo monopolio, sino también las PYMEs de la CAM.

Peso regional de la industria electrónica. El sector electrónico de Madrid supone 11.235 puestos de trabajo (1996). Con fluctuaciones, la participación el sector electrónico en el valor de la producción manufacturera de Madrid ha aumentado del 4,9\% en 1986 al 5,6\% en 1996. Por el contrario, la participación sectorial en el empleo industrial se ha reducido sensiblemente, del $7,1 \%$ al 4,5\%, dado que la caída del empleo sectorial ha sido aún más severa que la del empleo manufacturero regional durante ese período.

\footnotetext{
9 2,3 millones de clientes en España.

10 Con unas rentas anuales de 15.000 millones de dólares, Telefónica es el $14^{\circ}$ operador europeo de servicios de telecomunicaciones. Sus beneficios han sido de 1.309 millones de euros en 1998. Telefónica es el operador de servicios más rentable de la UE, seguido de Deutsche Telekom y de France Telecom (1998).

11 Esta expansión ha proseguido, a través de Telefónica Internacional (Tisa), en Suiza, Polonia, Hungría, Austria y la República Checa.
} 
Una de las razones de la caída del empleo en la industria electrónica de Madrid ha sido el rápido crecimiento de la productividad por asalariado (valor añadido /numero de asalariados) cuya tasa ha sido mucho más dinámica, además, que la de la industria madrileña en su conjunto (figura 1). El excedente bruto por asalariado de la electrónica, que al comienzo del período era menos de un tercio del de la industria regional, ha superado ampliamente a éste al final del período. Estos datos muestran el potencial de las empresas electrónicas de la región, incluso durante un período, no sólo de crisis internacionales del sector, sino también de apertura de la industria española, hasta entonces muy protegida de la concurrencia internacional.

Con Cataluña y el País Vasco, la CAM es una de las tres grandes Comunidades Autónomas españolas productoras de electrónica en España. Hay una cierta especialización regional. La producción madrileña de circuitos impresos, de equipos de telecomunicaciones y de productos electrónicos de consumo fue de 1.728,2 millones de ecus (46\% de la producción nacional) en 1996. La producción regional de ofimática (incluidos ordenadores), productos de óptica y de control fue de $1.321,3$ millones de ecus (22,9\% de la producción nacional) ${ }^{12}$. Madrid representa el 13\% del empleo, el 13,7\% de la producción y el $16,4 \%$ del valor añadido de la industria nacional de electrónica profesional (sanidad, defensa, robótica) y de componentes electrónicos (Hidalgo Nuchera, 1997). Pero la posición de la región es sobre todo incuestionable en manufacturas de equipos de telecomunicaciones. El índice de especialización (IS) de Madrid es 3,5 veces la media nacional en electrónica (excepto ordenadores) y 1,5-2,5 veces en ordenadores ${ }^{13}$ (Espina, 1994).

Las competencias tecnológicas están, incluso, muy concentradas en la CAM en lo que respecta a las comunicaciones y las telecomunicaciones, es decir los sectores dominantes de la electrónica regional. En su estudio de 3.810 patentes europeas depositadas por residentes en Es-

12 Esta categoría estadística incluye productos no electrónicos (INE, 1997; CAM, 1997).

${ }_{13}$ Calculado como: $\mathrm{IS}=\frac{V A E M / V A I M}{V A E E / V A I E} \quad$ y siendo VAEM el valor añadido de la in-

dustria electrónica de Madrid, VAIM el valor añadido de la industria de Madrid, VAEE el valor añadido de la industria electrónica española y VAIE el valor añadido de la industria española. 


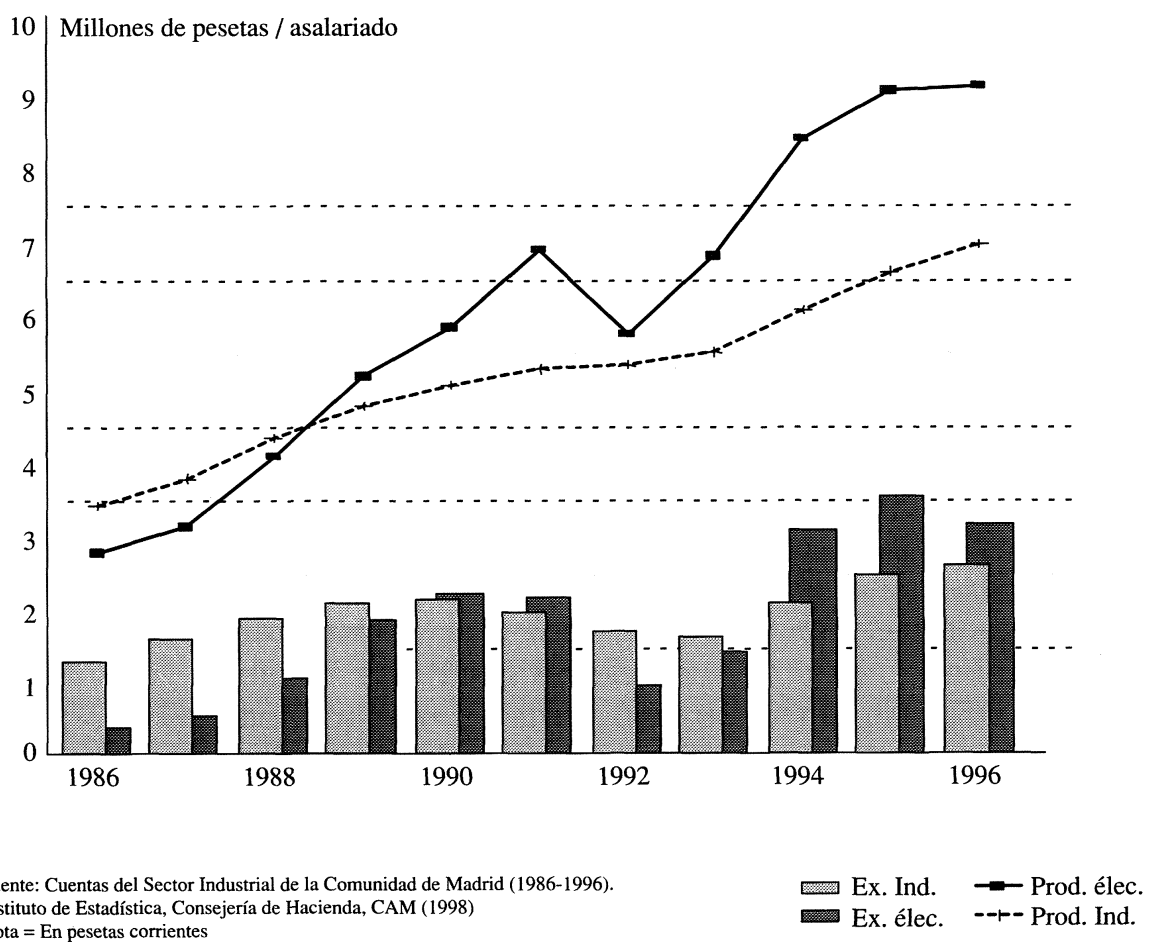

Fig. 1. Evolución de la productividad media y del excedente bruto por asalariado en la industria (total) y en el sector electrónico de la CAM (1986-1996).

paña en el período 1978-97, Sanz Menéndez y Arias (1998) identifican mediante un índice de ventajas tecnológicas reveladas, una fuerte especialización tecnológica de la CAM en electrónica, telecomunicaciones y audiovisual. Por el contrario, otras regiones productoras, como Cataluña y el País Vasco presentan una menor especialización tecnológica en electrónica.

Los análisis regionales sobre innovación, realizados a partir de datos de gastos en I+D confirman esos resultados. La Encuesta de Empresas Innovadoras del INE (INE, 1997) permite constatar que las firmas de la CAM hacen el $14,5 \%$ de la I+D nacional en ofimática y ordenadores, el $21 \%$ en circuitos impresos y el $84 \%$ en aparatos de radio, de televisión y de comunicaciones. 
Naturaleza y localización de los establecimientos electrónicos de la CAM

Número y localización de los establecimientos electrónicos. La comparación de los datos sectoriales revela que, entre 1988 y 1992, se produjo una severa reducción en el número de establecimientos, seguida de un repunte general desde esa fecha a 1998 (cuadro 1). No obstante, si se considera todo el período $1988-98$, y pese a un incremento del número total de establecimientos, se constata una reducción drástica de los establecimientos medios que contaban con 20 a 99 trabajadores. Este tipo de plantas, así como —en el período inicial 1988-92los pequeños establecimientos de menos de 20 trabajadores, han resultado los más frágiles durante la década de los noventa. La demografía industrial de Madrid podría haberse visto influenciada negativamente por la crisis de sobreproducción de circuitos impresos y por la subsecuente caída de sus precios (Martínez Romero, 1998; Rama, 1999). Se trata de un sector muy volátil en el que los pequeños establecimientos surgen en los períodos de expansión y desaparecen durante las crisis.

Cuadro I

NÚMERO DE ESTABLECIMIENTOS SEGÚN TAMAÑO EN LA INDUSTRIA ELECTRÓNICA DE LA CAM (1988-1998)

\begin{tabular}{|c|c|c|c|c|c|c|c|c|}
\hline \multirow[b]{2}{*}{$\begin{array}{c}\mathrm{N}^{\circ} \text { asala- } \\
\text { riados }\end{array}$} & \multirow[b]{2}{*}{1988} & \multirow[b]{2}{*}{1990} & \multirow[b]{2}{*}{1992} & \multirow[b]{2}{*}{1998} & \multicolumn{4}{|c|}{ Porcentaje de variación } \\
\hline & & & & & $\begin{array}{l}1992 / \\
1988\end{array}$ & $\begin{array}{l}1998 / \\
1992\end{array}$ & $\begin{array}{l}1998 / \\
1990\end{array}$ & $\begin{array}{l}1998 / \\
1988\end{array}$ \\
\hline 0 a 19 & 180 & 95 & 147 & 230 & $-18,3$ & 56,5 & 142,1 & 27,8 \\
\hline 20 a 49 & 57 & 29 & 45 & 47 & $-21,1$ & 4,4 & 62,1 & $-17,5$ \\
\hline 50 a 99 & 18 & 22 & 10 & 9 & $-44,4$ & $-10,0$ & $-59,1$ & $-50,0$ \\
\hline $100-499$ & 20 & 12 & 5 & 22 & $-75,0$ & 340,0 & 83,3 & 10,0 \\
\hline Más de 500 & 9 & 7 & 9 & 18 & 0,0 & 100,0 & 157,1 & 100,0 \\
\hline Total & 284 & 165 & 216 & 326 & $-23,9$ & 50,9 & 97,6 & 14,8 \\
\hline
\end{tabular}

Fuente: Elaboración propia con datos de Directorio Industrial CAM, 1988 y 1992; Directorio de Unidades. Locales 1998. CAM. 
Localización de los establecimientos electrónicos según su tamaño. El pequeño establecimiento electrónico es especialmente representativo de los espacios intrametropolitanos del Centro de Madrid, mientras que los de mayor tamaño están, por lo general, concentrados en los cinturones industriales que rodean la ciudad (cuadro II) ${ }^{14}$.

Se trata de un sector en el que algunos establecimientos grandes agrupan una parte sustancial del empleo. Por ejemplo, en equipo de telecomunicaciones, los establecimientos con más de 500 asalariados suponen el 90\% del empleo del subsector (Hidalgo Nuchera, 1997).

CUADRo II

DISTRIBUCIÓN GEOGRÁFICA DE ESTABLECIMIENTOS Y EMPLEOS EN LA INDUSTRIA ELECTRÓNICA DE LA CAM (1998)

\begin{tabular}{|c|c|c|c|c|c|}
\hline Localización & $\begin{array}{l}\text { Estable- } \\
\text { cimientos }\end{array}$ & Empleos & $\begin{array}{c}\text { Empl/ } \\
\text { Establec. }\end{array}$ & \% Establec. \% & 6 Empleos \\
\hline Municipio de Madrid & 156 & 3.037 & 19 & 47,85 & 28,95 \\
\hline - Almendra Central & 56 & 731 & 13 & 17,18 & 6,97 \\
\hline - Periferia Noroeste & 49 & 967 & 20 & 15,03 & 9,22 \\
\hline - Periferia Este & 18 & 1.028 & 57 & 5,52 & 9,80 \\
\hline - Periferia Sur & 33 & 311 & 9 & 10,12 & 2,96 \\
\hline Corona Metropolitana & 134 & 5.565 & 42 & 41,10 & 53,05 \\
\hline - Norte & 30 & 1.954 & 65 & 9,20 & 18,63 \\
\hline - Este & 40 & 1.618 & 40 & 12,27 & 15,42 \\
\hline - Sur & 54 & 1.888 & 35 & 16,56 & 18,00 \\
\hline - Oeste & 10 & 105 & 11 & 3,07 & 1,00 \\
\hline Total Zona Metropolitana & 290 & 10.010 & 35 & 88,96 & 95,42 \\
\hline Municipios no metropolitanos & 27 & 481 & 18 & 8,28 & 4,58 \\
\hline Total CAM & 326 & 10.491 & 32 & 100,00 & 100,00 \\
\hline
\end{tabular}

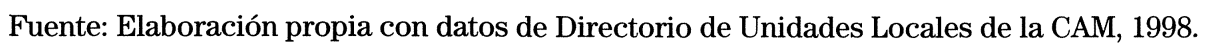

Ampliación de la base geográfica de la industria electrónica de la Región. Los establecimientos electrónicos están, principalmente, concentrados en la zona central (figura 2) aunque, desde el punto de vista del empleo la concentración sectorial está mucho menos marcada, dado el gran tamaño de los establecimientos de la periferia.

${ }^{14}$ Los últimos datos disponibles clasificados por municipios datan de 1992. 


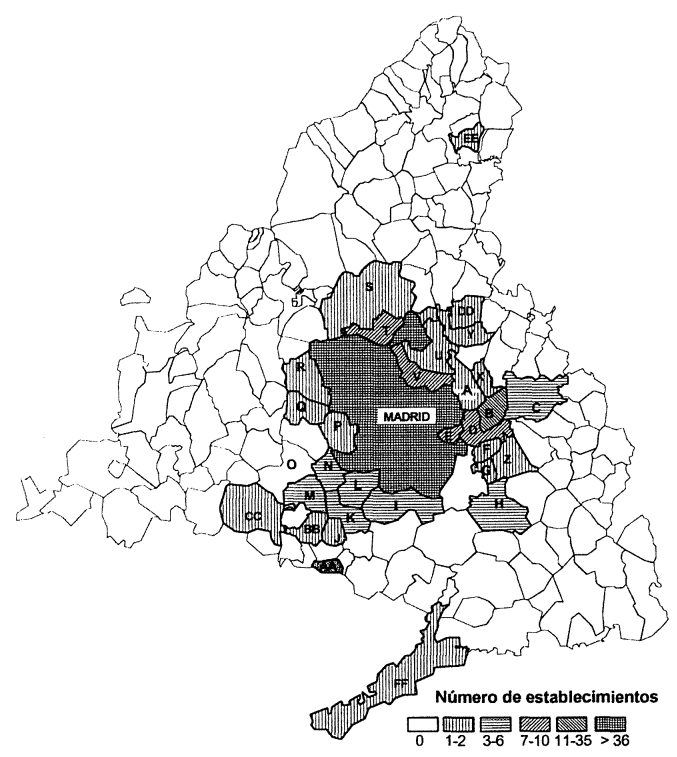

A Paracuellos del Jarama

B Torrejón de Ardoz

C Alcalá de Henares

D San Fernando de Henares

E Coslada

F Mejorada del Campo

G Velilla de San Antonio

H Arganda del Rey

I Getafe

$J$ Humanes

K Fuenlabrada

L Leganés

$M$ Móstoles

N Alcorcon

N Alcorcon

P Pozuelo de Alarcón

Q Majadahonda

R Las Rozas

S Colmenar Viejo

T Tres Cantos

U San Sebastián de los R.

$\mathrm{V}$ Alcobendas

$\mathrm{X}$ Ajalvir

$\mathrm{Y}$ Algete

$Z$ Loeches

AA Cubas

BB Moraleja de Enmedio

CC Navalcarnero

EE Robledillo de la Jara

FF Aranjuez

Fig. 2. Distribución de los establecimientos de la industria electrónica en la Comunidad de Madrid, 1992.

Probablemente, han sido los establecimientos de gran tamaño los responsables de la ampliación de la base geográfica de esta industria durante el último período, ya que se han instalado, sobre todo, en la periferia, especialmente en las nuevas zonas industriales y en el Parque Tecnológico de Madrid (PTM) ubicado en el municipio de Tres Cantos.

Instalado en 1985, este Parque de la periferia norte de Madrid, ha constituido un polo de atracción para cierto número de empresas del sector. Uno de los criterios de aceptación de las firmas ha sido que estuvieran introducidas en microelectrónica, informática, telecomunicaciones, automatización, láseres, nuevos materiales, biotecnología y otros proyectos de fuerte contenido innovador (IMADE, 1995). Aunque el Parque no sea comparable en términos de especialización con los polos tecnológicos franceses tiene, en cambio, una vocación claramente orientada hacia la electrónica. Los resultados de la encuesta de MellaMárquez y Álvarez-González (1998) revelan que al menos el 78\% de las 
empresas manufactureras del PTM están directamente ocupadas en la producción de productos electrónicos (36\% en telecomunicaciones, $21 \%$ en ingeniería electrónica y $21 \%$ en informática). A ello, hay que añadir la presencia de firmas de la industria aerospacial, entre las que cabe citar a Alcatel Spatiale (sobre la importancia de las compañías aerospaciales en la formación de sistemas productivos industriales (clusters) informáticos, ver Gray et al., 1996). En el Parque, el sector aerospacial es el que cuenta con mayor número de firmas (Ondategui Rubio, 1998). Todo ello, sin hablar de las firmas de servicios, de las cuales el $28 \%$ son firmas de comunicación, el $7 \%$ de control de redes electrónicas y algunas otras de $\mathrm{I}+\mathrm{D}$ y servicios técnicos ligados a la industria electrónica (Mella-Márquez y Álvarez-González,1998). Finalmente, en su análisis de los microentornos innovadores de la Región madrileña, Cuadrado Roura et al. (1995) señalan el liderazgo de Telefónica como comprador de bienes y servicios en lo que ellos llaman el «subsistema» de Tres Cantos. Todo confirma, por tanto, la vocación electrónica del Parque, aunque estén allí presentes otras industrias.

\section{Identificación de redes}

Con relación a otras regiones españolas, como Cataluña, Valencia, País Vasco, La Rioja y las Islas Baleares, en las que la importancia de los lazos de cooperación entre empresas es conocida, Madrid nunca se había considerado como una región en la que podría desarrollarse una cultura de la cooperación de larga duración (Espina, 1994, p. 154).

En este apartado, trataremos de demostrar la existencia de dos polos en la industria electrónica de la región. Esos polos se sitúan en un tejido industrial donde las relaciones locales son muy significativas. Los grandes clientes de los principales subsectores regionales, como el equipo de telecomunicaciones y de comunicaciones al igual que el del audiovisual, se encuentran en Madrid (operadores de servicios, televisiones, comunicaciones por satélite). Además, alrededor del $52 \%$ de los establecimientos electrónicos adquieren más de la mitad de sus inputs en la región (Suárez-Villa y Rama, 1996).

La bipolarización de la región dimana, en nuestra opinión, de la existencia, por una parte, de un núcleo de pequeños y medianos establecimientos ligados por redes de naturaleza más bien igualitaria, don- 
de el liderazgo no está definido y en los que una buena parte de las unidades son simultáneamente dadores de órdenes (DO) y tomadores de órdenes (TO) ${ }^{15}$. Ese polo está especialmente localizado en los distritos del Centro, en el medio urbano. En los municipios periféricos del Norte y del Noreste, principalmente, encontramos, por el contrario, un grupo de establecimientos de mayor tamaño, en los que una buena parte son filiales de empresas multinacionales o de grandes grupos nacionales de electrónica. Estos establecimientos están sobre todo integrados verticalmente o desarrollan relaciones con su casa matriz (en España o en el extranjero). Algunos de ellos se integran en redes a nivel supra regional, e incluso nacional o internacional. En cambio, se integran mucho menos en redes regionales, con la excepción de los establecimientos del PTM que parecen cooperar sobre todo entre ellos.

En esta parte, examinaremos en esos dos grupos de establecimientos, lo que es la $\mathrm{I}+\mathrm{D}$, las relaciones con sus clientes y proveedores y la subcontratación, el comportamiento cooperativo y la importancia de la proximidad. Trataremos también de evaluar las diferencias entre dichos grupos.

El esfuerzo tecnológico y el papel de la cooperación. Los datos disponibles no permiten distinguir substanciales diferencias en el esfuerzo innovador (porcentaje de los gastos en $\mathrm{I}+\mathrm{D}$ con relación a la cifra de negocios) de los pequeños establecimientos del Centro y de los grandes establecimientos de la periferia, lo que constituye un resultado bastante sorprendente dada la evidencia proporcionada por la teoría económica sobre la asociación positiva entre tamaño y esfuerzo innovador de las firmas (Gaffard, 199016; Acs e Isberg, 1991; Buesa y Molero, 1996). Sin embargo, conviene recordarlo, nosotras trabajamos a nivel de establecimiento, no a nivel de empresa. Un cierto número de grandes establecimientos de la periferia, sobre todo cuando se trata de filiales de empresas multinacionales o de grupos nacionales, no hacen ellos mismos I+D sino que pueden hacerla su casa matriz o laboratorios de su grupo (a veces situados fuera de la región). Eso explicaría que el esfuerzo innovador esté muy igualado en los dos tipos de establecimientos que

${ }^{15}$ Suárez-Villa y Rama (1996) y primeros resultados de la encuesta 1999 del IEGCSIC

${ }_{16}$ Uno de los argumentos habitualmente esgrimidos es que únicamente las firmas que obtienen un cash flow importante, que son generalmente de gran tamaño pueden financiar un intenso esfuerzo en $\mathrm{I}+\mathrm{D}$. 
analizamos en este trabajo. Aquí, compararemos principalmente los resultados encontrados en el municipio de Tres Cantos (una de las implantaciones especializadas más avanzadas de la industria electrónica regional), con los datos generales sobre el esfuerzo innovador en ese sector madrileño.

Ondategui Rubio (1998), que realizó, en 1995, entrevistas en 120 empresas del municipio de Tres Cantos afirma que, en las de software, el $50 \%$ de los empleados se dedican al desarrollo y a la adaptación de productos; en telemática y multimedia encontró un porcentaje inferior, aunque no precisa la participación. Finalmente, en las restantes firmas de electrónica visitadas por este autor, alrededor del $25 \%$ de los asalariados se dedican a actividades de I+D. Encontró, también, que las firmas de las empresas aerospaciales y de «software» de este municipio destinaban a I+D del 10 al 15\% de sus cifras de negocio; en cambio, el resto de las empresas de la industria electrónica, canalizaban a las actividades innovadoras entre el 6 y el $8 \%$ de las suyas. Hay que señalar que esas cifras podrían sobrevalorar el esfuerzo innovador de la electrónica de Tres Cantos habida cuenta de la presencia de ATT en el PTM. Instalada en la región desde 1989, la multinacional americana emplea a 760 personas; el $98 \%$ de su producción se destina al mercado europeo de circuitos impresos. La fábrica de Tres Cantos utiliza una tecnología y alcanza niveles de desempeño económico que, en 1995, eran similares a los de la casa matriz en Estados Unidos (Ondategui Rubio, 1998).

En la encuesta llevada a cabo en 1993 en 64 establecimientos de la Comunidad de Madrid, incluidos los distritos intra-metropolitanos, Suárez-Villa y Rama (1996) encuentran que la media de los gastos en I+D respecto a la cifra de negocios es del $8,4 \%$, es decir, comparable muy favorablemente con las cifras proporcionadas por Ondategui Rubio (1988) sobre los establecimientos (excepto «software») de una de las más importantes localizaciones electrónicas de la periferia.

Si bien la dimensión del esfuerzo innovador es comparable entre los dos núcleos, la organización de la I+D es muy distinta. Mientras que los establecimientos de Tres Cantos apuestan principalmente por asociaciones externas con centros especializados (universidades locales o interlocutores europeos ${ }^{17}$ ), los pequeños establecimientos del

\footnotetext{
${ }^{17}$ Se puede decir que la participación de empresas electrónicas españolas en los programas tecnológicos de la UE ha sido sustancial, aunque no disponemos de datos por regiones. Actualmente, la participación española acumulada en el Programa ESPRIT
} 
Centro prácticamente no tienen relaciones con departamentos universitarios ${ }^{18}$.

Los establecimientos de equipo de telecomunicaciones, de «software» y de otros bienes de equipo con fuerte contenido electrónico del Parque Tecnológico tienen conexiones importantes con centros de investigación y Universidades locales (Ondategui Rubio, 1988). Su estrategia sería fomentar la realización de invenciones radicales con socios externos a la fábrica ${ }^{19}$. Hay tres empresas creadas por «spin-offs» procedentes de proyectos de Universidades de la región. La administración, a través del CDTI (Centro para el Desarrollo Tecnológico Industrial), proporciona a las firmas electrónicas recursos reembolsables sin interés para desarrollar proyectos de cooperación precompetitivos con centros públicos de investigación, aunque el número de proyectos financiados por este concepto sea muy limitado (Hidalgo Nuchera, 1997) ${ }^{20}$. Los vínculos tecnológicos con las casas matriz son, asimismo, intensos en este grupo. Por otra parte, estos establecimientos, desarrollan gran número de proyectos con socios europeos ${ }^{21}$.

Otra cosa muy distinta son las relaciones inter-firmas. La encuesta de Mella-Márquez y Álvarez-González (1998) muestra que las empresas del Parque Tecnológico (electrónicas y otras) mantienen, primero, vínculos de cooperación tecnológica con otras compañías del Parque y, después, con empresas extranjeras; paradójicamente, la cooperación con compañías de la CAM (excluido el Parque) y el resto de España es menos significativa.

En general, los pequeños establecimientos urbanos no mantendrían acuerdos específicos para la cooperación en $\mathrm{I}+\mathrm{D}$, pero utilizarían las re-

(electrónica) de la UE representa cerca del 28\% de los proyectos. En el Programa ACTS (multimedia, redes de comunicaciones, etc.) se eleva al 35\% en 1994-98. En 1995, esos dos Programas junto con el de Aplicaciones Telemáticas han proporcionado 34,5 millones de ecus a proyectos con participación española (Hidalgo Nuchera, 1997).

${ }_{18}$ Resultados de la encuesta de 1993 de Suárez-Villa y Rama.

19 Van het Kaar y Kok (1996) ponen de manifiesto que de 62 proyectos contratados entre las firmas del Parque y las Universidades de la CAM en el período 1989-96, 39 de ellos concernían a la electrónica. Los proyectos electrónicos, que representaron el $97 \%$ de todos los proyectos firmados con la Universidad Politécnica de Madrid, se refieren a radares, transmisión de datos, radiocomunicaciones, microondas, audiovisual, electroacústica, inteligencia artificial, etc.

${ }_{20}$ En toda España, solamente 17 proyectos en 1996 (Hidalgo Nuchera, 1997).

${ }^{21}$ En 1995, las compañías que habían establecido partenariados europeos para hacer I + D conjunta representaban el 58\% de las empresas de Tres Cantos (electrónicas y otras) (Ondategui Rubio, 1998). 
des de subcontratación como un medio para especializarse y para dedicar más recursos internos a las tareas de innovación (Suárez-Villa y Rama, 1996). En este contexto, la solución de problemas en común entre el DO y el subcontratante es también una herramienta para la transmisión de técnicas nuevas (Freeman, 1991; Cooke y Morgan, 1993).

Proveedores. Los establecimientos pequeños del Centro tienen tendencia a aprovisionarse en la Región, mientras que los de la periferia dependerían más de proveedores extra-regionales, incluso nacionales o extranjeros. Mella-Marquez y Álvarez-González (1988) indican que, entre los abastecedores de las empresas del Parque, el 79\% son firmas extranjeras, el $21 \%$ son empresas nacionales de fuera de la región y únicamente el $14 \%$ son compañías de la CAM.

Este comportamiento de las firmas de alta tecnología de Tres Cantos es muy diferente del que presenta el conjunto de empresas electrónicas de la CAM que están mucho más orientadas a la propia región. Suárez-Villa y Rama (1996) observan diferencias importantes de comportamiento para el aprovisionamiento, según el tamaño de los establecimientos. Son sobre todo las fábricas pequeñas - hay que recordar que éstas están principalmente concentradas en el medio urbano- las que tienden a abastecerse en la CAM. Si comparamos dos extremos, se constata que cerca del $62 \%$ de los establecimientos pequeños (menos de 50 empleados) adquieren más de la mitad de sus inputs en la región. En cambio, en el segmento de los establecimientos grandes (más de 500 empleados), únicamente el $14 \%$ se encuentra en esa situación. Inversamente, los establecimientos pequeños importan sólo el $15 \%$ de sus inputs, mientras que los establecimientos grandes, cuyos vínculos con el mercado internacional son mucho más considerables, importan el 47\% (Suárez-Villa y Rama, 1996).

Por subsectores, las diferencias de comportamiento según tamaño son también evidentes. Una encuesta que incluía 34 establecimientos madrileños de equipo para telecomunicaciones, el sector más importante de la industria electrónica regional, subraya, igualmente, su propensión a abastecerse en la Región (el 34,4\% de los establecimientos adquieren en ella más de la mitad de sus inputs) (Rama, 1992). Sin embargo este trabajo también muestra diferencias de comportamiento entre pequeños y grandes establecimientos ${ }^{22}$. Estos últimos que, según los resultados de

${ }^{22}$ Los grandes establecimientos están definidos en la encuesta de establecimientos de equipo para telecomunicaciones como los que cuentan con más de 500 empleados. 
un test estadístico «t», tienden a ser filiales de empresas multinacionales o empresas conjuntas (joint-ventures), compran en la región menos de la cuarta parte de sus inputs. En cambio, las PYMEs adquieren en ella el $52 \%$ del valor de los suyos. En el subsector de la electrónica profesional y de los componentes, los establecimientos madrileños de menos de 20 empleados efectúan el 74,1\% de sus compras en la región, en 1995, mientras que los de más de 20 empleados solamente realizan en ella un tercio de las suyas (Hidalgo Nuchera, 1997). Conviene señalar, una vez más, que los pequeños establecimientos están ubicados, por lo general, en la zona intra-metropolitana de la CAM.

La propia percepción de los establecimientos en cuanto a la importancia de la proximidad de los proveedores varía mucho en función de su localización central o periférica. Murillo Castillejo (1997), que estudia los factores de localización de 42 establecimientos electrónicos de Madrid de reciente instalación o desplazados en el período 1980-91, encuentra que la proximidad de los proveedores es crucial para los establecimientos del centro, mientras que ese factor de proximidad no se cita por los establecimientos de la periferia, los cuales valoran especialmente la calidad del entorno residencial y la disponibilidad de espacio industrial. Van het Kaar y Kok (1996) confirman la supremacía de otros factores de localización industrial, diferentes de la proximidad de los abastecedores, entre las empresas del PTM. Las firmas (electrónicas y otras) entrevistadas por estos autores declaran que los motivos más decisivos para llegar a instalarse en el Parque habrían sido, por orden de importancia, la presencia de compañías similares, la proximidad de la metrópoli y los bajos costes de instalación (dadas las subvenciones y las ayudas a las empresas de la Comunidad Europea). A estos motivos les siguen la imagen de prestigio del Parque y la proximidad de numerosas Universidades y Centros de Investigación. En cambio, la proximidad de los abastecedores no es siquiera citada.

Clientes. Según Mella-Márquez y Álvarez-González (1988), la vocación extra-regional de las firmas del Parque ${ }^{23}$ es también evidente respecto a la distribución geográfica de sus clientes, de los cuales el $67 \%$ procede del resto de España (fuera de la región), el 27\% del extranjero y, únicamente, el 7\% de la Región de Madrid. Estos datos deben compararse con los del destino de la producción de la industria electrónica de Ma-

${ }^{23}$ Incluidas firmas no electrónicas. 
drid en su conjunto que está situado principalmente en la propia región (41,4\%), seguido del resto de España (34,5\%), países no europeos $(19,4 \%)$ y, finalmente, Europa (4,7\%) (CAM, 1992).

En el subsector madrileño de equipo de telecomunicaciones, se observan, igualmente, grandes diferencias entre establecimientos según su tamaño (y, probablemente, su localización espacial) (Rama, 1992). Los pequeños establecimientos de menos de 100 empleados realizan principalmente su cifra de negocios en la Región (56,6\% de la cifra de negocios total) o en el resto del país (34,2\%), mientras que sus exportaciones son modestas (4,6\%). Los grandes establecimientos de más de 100 empleados realizan también en la Región una parte considerable (50,2\%) de su cifra de negocios, ya que en ella se encuentran los grandes compradores, principalmente la sede de Telefónica. Sin embargo, su proyección nacional y, especialmente, exportadora son mucho más significativas, ya que realizan con el resto de España el 36,6\% de su cifra de negocios, y el 14,2 \% con el extranjero.

\section{Subcontratación ${ }^{24}$}

En términos generales la subcontratación es muy importante en la industria electrónica de Madrid. La encuesta de 1993, mostraba que el 81 $\%$ de los establecimientos estaban comprometidos en ese tipo de actividades como $\mathrm{DO}$ o TO; el $68 \%$ de los establecimientos formaba redes particularmente complejas, por su compromiso en la subcontratación «a dos vías» (eran simultáneamente DO y TO). Los resultados preliminares de la encuesta de 1999 parecen confirmar aquellos resultados.

El análisis estadístico de Suárez-Villa y Rama (1996) identifica las redes más densas de subcontratación en la industria electrónica del Centro, independientemente incluso del tamaño del establecimiento ${ }^{25}$. En los tests de asociación entre las variables tamaño e $I+D$ se aprecia que las correlaciones estadísticas son más estrechas en el caso de los es-

24 Aquí consideramos únicamente la subcontratación entre establecimientos electrónicos, a diferencia del análisis de las Comunidades Europeas, que considera también subcontratantes de sectores de la metalúrgia (placas, etc.), del caucho y de los plásticos (cajas de ordenadores, etc.) de la industria electrónica (CE, 1997).

${ }_{25}$ No obstante, los establecimientos pequeños se mostraban más inclinados que los grandes a estar comprometidos en actividades de subcontratación. 
tablecimientos electrónicos de esos distritos que entre los de la periferia. Estos resultados se han interpretado como indicativos de una adaptación especialmente atinada entre el capital invertido y el esfuerzo innovador del establecimiento. Las posibilidades de subcontratación ofrecidas por la localización en la ciudad de Madrid han permitido a los establecimientos mantener intra-muros únicamente las tareas especializadas que se relacionaban con su esfuerzo innovador y aceptar ordenes que tenían justamente relación con sus competencias. En cambio, el resto de actividades productoras para las que los establecimientos no poseían competencias técnicas especiales eran subcontratadas a otros establecimientos . Esto ha permitido profundizar en la especialización de las fábricas y desarrollar la división del trabajo en el seno de la red. La organización en red de los pequeños establecimientos intra-metropolitanos, por un lado, y la dependencia de las filiales de la casa matriz para la innovación entre los establecimientos de gran tamaño, por otro, explicarían, por tanto, la paradoja de la similitud del esfuerzo innovador.

Al mismo tiempo, los tests estadísticos de la asociación de la variable independiente capital (calculada por tres fórmulas distintas) y los gastos en $I+D$ presentan un coeficiente más elevado en la categoría de los establecimientos electrónicos del Centro que estaban comprometidos en la subcontratación a dos vías ${ }^{26}$. Dado el acortamiento del ciclo de vida del producto en la industria electrónica mundial y su efecto devastador sobre la obsolescencia de las inversiones de las empresas (Ernest, 1998), es muy importante, desde el punto de vista de la competitividad, que las firmas no hagan más que un mínimo de inversiones y que éstas tengan relación con las tareas en las que aquellas estén directamente comprometidas a tiempo completo. La organización en red ha permitido a los pequeños establecimientos del Centro no adquirir el equipo del que hacían sólo un uso esporádico. Es evidente que esta estrategia minimiza los riesgos asociados con la rápida obsolescencia que es intrínseca a la industria electrónica, dado el ritmo de cambio tec-

\footnotetext{
${ }^{26}$ Las seis categorías de establecimientos testados han sido las siguientes:

A) En los municipios del Centro: todos los establecimientos; establecimientos comprometidos en actividades de subcontratación; y establecimientos comprometido en la subcontratación a dos vías.

B) En los municipios de la periferia: todos los establecimientos; establecimientos comprometidos en actividades de subcontratación; y establecimientos comprometido en la subcontratación a dos vías.
} 
nológico que la caracteriza. Igualmente, gracias a las redes de contratación, las PYMEs del Centro han podido beneficiarse colectivamente de la tecnología más avanzada concerniente a cada fase del proceso productivo, incluso si esos activos no eran propiedad más que de algunas de ellas.

El trabajo citado pone igualmente a prueba la hipótesis de la asociación estadística entre los costes del establecimiento (desagregados en costes de los materiales y de transportes) y la $I+D$; poniendo en evidencia que el coeficiente más alto es el de los establecimientos del Centro, lo que indica mayor eficacia de los desembolsos efectuados por esos establecimientos que, como se ha visto, tienden a participar más en las redes de subcontratación. Es decir que esos establecimientos aciertan al racionalizar más sus gastos, con lo que pueden apoyar más eficazmente el desarrollo de nuevos productos (equipamiento de usos múltiples, por ejemplo).

Los resultados del test demuestran la funcionalidad de las redes para las PYMEs ya que, en términos de valor absolutos, los costes unitarios por empleado de los establecimientos del centro eran un $4 \%$ superiores a los de los establecimientos de la periferia, los cuales se benefician, naturalmente, de economías de escala debido a su mayor tamaño.

\section{Conclusiones}

En este apartado trataremos, en primer lugar, de destacar las principales conclusiones de nuestro trabajo de investigación y, posteriormente, de reflexionar sobre lo que este estudio de caso de la Comunidad de Madrid puede indicarnos, en términos más generales, acerca de las redes de empresas.

Los comportamientos de los pequeños y medianos establecimientos electrónicos, generalmente localizados en el Centro, y los grandes establecimientos, predominando en los cinturones industriales y el Parque Tecnológico, son diferentes en lo concerniente a sus relaciones de cooperación en $\mathrm{I}+\mathrm{D}$, así como en las relaciones que mantienen con sus clientes, sus proveedores y sus subcontratantes. La localización intrametropolitana está asociada a la estructuración de redes bastante densas de subcontratación y a relaciones con proveedores y clientes de la región. La cooperación para la innovación emana, en este caso, de un es- 
fuerzo de racionalización de costes y de inversiones llevado a cabo por el establecimiento a través de la formación de redes locales de subcontratación, que facilitan la especialización de tareas, así como la cooperación técnica entre DO y TO. En este núcleo, la participación de los establecimientos en redes complejas y relativamente estables de subcontratación les ha permitido la conjunción de un aprendizaje acumulado y de activos físicos específicos (Monateri y Ruffieux, 1996, p. 105).

En cambio, entre los grandes establecimientos de los municipios periféricos, es la cooperación dentro del grupo o con Universidades e Instituciones de investigación pública, así como la participación en proyectos de la UE, lo que juega el papel más importante en lo concertiente a la cooperación en I+D. La formación de redes de empresas, cuando existe, no es local.

Sin embargo, es posible una interpretación alternativa del fenómeno observado, que habría que explorar más adelante. Los establecimientos electrónicos de la periferia podrían integrarse, en cierta medida, en redes formadas por unidades localizadas en determinadas provincias limítrofes de Madrid, principalmente Toledo y Guadalajara. Debido a que estas regiones han sido consideradas por la Unión Europea dentro del Objetivo $1^{27}$ y habida cuenta de la activa promoción de sus zonas industriales, ambas provincias han atraído, durante los últimos años, numerosas inversiones, de las cuales una gran parte de origen madrileño (Celada y Méndez, 1994). La Comunidad de Madrid se extiende a lo largo y ancho de un territorio reducido (escasamente $8.000 \mathrm{~km}^{2}$, lo que facilita los contactos con esas provincias. Estas «zonas de descongestión» de Madrid, están, por tanto, asociadas a la aparición de un «efecto frontera» significativo. Hay que señalar que esas provincias limítrofes subcontratan principalmente en la Comunidad de Madrid ${ }^{28}$. Entre las grandes empresas electrónicas con implantaciones en esas provincias se pueden mencionar, en Toledo, la fábrica de hardware de Alcatel ${ }^{29}$ y la de la multinacional alemana Siemens Nixdorf International. Entre las industrias conexas, se puede señalar, igualmente, la presencia de CASA,

\footnotetext{
${ }^{27}$ Su PIB no alcanza el 75\% del PIB comunitario.

${ }_{28}$ En su encuesta de 268 empresas de 43 municipios fronterizos de la Comunidad de Castilla-La Mancha (100 Km de distancia máxima de Madrid), Celada y Méndez (1994, p. 208) encuentran que el $43 \%$ de los DO (de todos los sectores) tenían su principal subcontratante en la CAM.

${ }^{29}$ La multinacional francesa tiene también otras tres filiales, una de ellas de software, en la CAM.
} 
empresa pública española (7.600 empledos) del sector aeronáutico que obtiene el $80 \%$ de su cifra de negocios mediante la exportación. CASA tiene 290 subcontratantes, de ellos 225 (entre los que hay numerosas firmas de software) en la CAM (Fernández, 1998).

Si esta hipótesis se revelara exacta, la bipolaridad de Madrid tendría, entonces, relación con dos tipos de polos electrónicos: uno intra metropolitano y otro que abarcaría una dimensión de la región más amplia que la que definen los límites políticos de la Comunidad de Madrid.

La formación de redes complejas en las que la proximidad tiene un papel decisivo puede interpretarse como un fenómeno de racionalización sistémica por parte de los establecimientos del Centro. Esas redes, en gran medida, han permitido a las PYMEs electrónicas de la Región de Madrid sobrevivir a las numerosas y graves crisis y fluctuaciones que se han sucedido en ese sector a lo largo de los años noventa (Suárez-Villa y Rama, 1996) y beneficiarse de las nuevas oportunidades abiertas merced a la liberalización de los servicios de telecomunicaciones y a la expansión internacional de Telefónica. Reparando en el análisis de Lorenzoni (1982, citado por Jarillo, 1988, p. 32), que distingue tres etapas en la formación de redes de PYMEs, llegamos a la conclusión de que las PYMEs intra metropolitanas de Madrid han superado, a partir de los años 80 , la fase de «reacción», durante la cual han actuado, principalmente, como proveedores de las grandes firmas electrónicas, en productos intensivos en mano de obra (Estevan, 1988; Benton, 1990; Suárez-Villa y Rama, 1996). En el momento actual están, probablemente en la fase de «constelación racionalizada» (rationalized constellation), ya que no es evidente que hayan llegado aún a la tercera fase de «constelación planificada» (planned constellation).

Podemos decir que la formación de redes ha sido espontánea, es decir que no ha sido impulsada por los poderes públicos españoles quienes únicamente subvencionan la cooperación en $\mathrm{I}+\mathrm{D}$ con socios europeos ${ }^{30}$ sin prever ningún tipo de ayuda para la cooperación, sea cual fuere, entre socios regionales ${ }^{31}$. Esto desmentiría la afirmación de algunos au-

30 Principalmente las subvenciones de la UE, a través del CDTI .

31 En España no existen mecanismos de estimulación de la cooperación y de mediación institucional parecidos a los de Redes de Difusión Tecnológica (RDT) de la Agencia Nacional para la Valoración de la Investigación (ANVAR) de Francia (Filippi, 1996, p. 736). Tampoco parecen haber operado los mecanismos de estímulo de las organizaciones profesionales que describe Saxonian (1992) en Silicon Valey. 
tores de que la cooperación entre PYMEs no tendría lugar si no estuviera estimulada por los poderes públicos (ver, por ejemplo, Héraud et al., 1994, p. 13) ${ }^{32}$.

La formación de redes no es tampoco obra de un sustrato históricocultural ni se debe a la existencia de afinidades sociales, uno de los elementos clave de los que carece Madrid para definirlo como un distrito «canónico» (Becattini, 1990; Signorini, 1994; Suárez-Villa y Rama, 1996). Paradójicamente, es el municipio donde se encuentra el Parque Tecnológico el que empieza a beneficiarse de una identidad cultural similar que procede de la idea de una zona «moderna», de alta tecnología, de un microentorno de calidad, de una gran uniformidad del nivel cultural y de formación (Cuadrado et al., 1995, p. 11). No obstante, es cierto que es parecido el origen de un gran número de PYMEs del Centro en la medida en que fueron creadas por ingenieros que habían sido despedidos por las grandes empresas a consecuencia de las crisis del sector o que habían abandonado su trabajo voluntariamente. Esto podría haber estimulado relaciones de confianza mutua y una semejanza de valores y de motivaciones entre los empresarios. Lo anterior confirmaría el punto de vista de numerosos autores que piensan que esos elementos serían esenciales para el establecimiento de lazos de cooperación efectivos entre firmas (ver, por ejemplo, Jarillo, 1998, p. 37).

Considerando la inexistencia de antecedentes culturales de la cooperación en la CAM, este estudio de caso permite igualmente apreciar que las PYMEs son capaces de estructurar verdaderas estrategias, al igual que las grandes firmas, y que aquellas no se benefician exclusivamente de relaciones informales de entorno (de milieu) (Héraud y Nanopoulos, 1994, p. 6). En este sentido, las alianzas establecidas por las PYMEs intra metropolitanas serían asimilables a la definición de redes estratégicas de Jarillo (1998, p. 32), es decir que se trataría de organizaciones utilizadas deliberadamente por los empresarios para obtener una ventaja competitiva para sus firmas. Sin embargo, la gran diferencia con la definición de este autor es que él concibe esta organización como la obra de una firma líder, capaz de organizar la red en beneficio propio, mientras que los establecimientos electrónicos intra metropolitanos, debido a que muy frecuentemente están comprometidos a la vez

${ }^{32}$ «Entre PYMEs, en particular, no hay que contar demasiado con una tendencia espontánea a la cooperación» (Traducción de las autoras). 
como DO y TO en las relaciones de subcontratación, mantienen relaciones más igualitarias.

Los lazos entre los dos sistemas que coexisten en la electrónica madrileña se conocen insuficientemente, pero es probable que esos dos grupos de establecimientos ofrezcan productos diferentes y que la concurrencia tenga también lugar en «nichos» de mercado diferentes.

Por último, la comparación de datos de las dos encuestas citadas en este estudio (1993 y 1999), no proporciona elementos susceptibles de hacernos pensar que los establecimientos de Madrid estén internalizando sus funciones, a medida que se van familiarizando con las nuevas tecnologías, como sugiere Freeman (1991, p. 510). Por el contrario, los datos de 1999 nos inducen a considerar que no sólo las actividades de subcontratación continúan teniendo un gran peso en la CAM sino que también otros tipos de cooperación (I+D, marketing, formación temporal de uniones de empresas para un proyecto concreto, etc.), hasta ahora insuficientemente estudiados, son igualmente importantes.

\section{BIBLIOGRAFÍA}

Acs, Z. J. e Isberg, S. C. (1991): «Innovation, Firm Size and Corporate Finance: An Initial Inquiry», Economic Letters, 35, 323-326.

BecatTini, G. (1990): «The Marshallian District as a Socio-Economic Notion», en: P. Pyke, G. Becattini and W. Sengerberger (Eds.) Industrial Districts and Inter-Firm Cooperation in Italy, 37-51, Geneva: International Institute for Labour Studies.

Benton, L.(1990): Invisible Factories: The Informal Economy and Industrial Development in Spain, Albany, State University of New York Press.

Buesa, M. y Molero, J. (1996): Tamaño Empresarial e Innovación Tecnológica en la Economía Española, Document mimeo, Instituto de Análisis Industrial y Financiero, Universidad Complutense de Madrid.

Buesa, M. y Molero, J. (1998): «Madrid, Capital de la Innovación Tecnológica Española», Economistas, n. ${ }^{\circ} 79,88-98$.

Calatrava Andrés, A. y Melero Gullló, A. (1999): «Dificultades Metodológicas y Estadísticas en el Análisis Sectorial Regional: El Caso del Sector de fabricación de Productos Electrónicos en la Comunidad Autónoma de Madrid», comunicación a las Primeras Jornadas Valencianas de Estudios Regionales, Valencia, 9 y 10 de abril de 1999.

CELADA, F. y MÉNDEZ, R. (1994): «Difusión Metropolitana de la Industria y Efecto Frontera en la Comunidad de Madrid», Revista de Estudios Regionales de la Comunidad de Madrid, n. ${ }^{\circ} 11,197-217$

COMMUNAUTÉs EUROPÉENNES (1997), «La sous-traitance dans le secteur électronique», in: La nouvelle sous-traitance industrielle en Europe. Premiers résultats chiffrés avec une définition actualisée, DGXXIII.

COMUNIDAD DE MADRID (1992): Directorio Industrial de la CAM, Consejería de Economía de la Comunidad de Madrid, Madrid. 
COMUNIDAD DE MADRID (1997): Cuentas del Sector Industrial de la Comunidad de Madrid1996 (base 96), Instituto de Estadística, Consejería de Hacienda de la CAM, Madrid.

CoOKe, P. y Morgan, K. (1993): «The Network Paradigm: New Departures in Corporate and Regional Development», Environnment and Planing D: Society and Space, Vol. 11, 543-564.

Cuadrado Roura, J. M. y Martínez Sierra, F. J. (1995): Dinámica Urbana e Innovación en el Área Metropolitana de Madrid: Análisis de Tres Subsistemas Específicos, Departamento de Economía Aplicada, Universidad de Alcalá, Madrid.

DELGADO RodRíGuez, M. ${ }^{a}$ J. (1998): «Las Infraestructuras Madrileñas», Economistas, n. ${ }^{\circ}$ 79, $72-87$.

ERNEST, D. (1998): «Cartching up, Crisis and Truncated Industrial Upgrading. Evolutionary Aspects of Technological Learning in East Asia's Electronics Industry», International Conference The Economics of Industrial Structure and Innovation Dynamics, INTECH-European Commission, Lisbon, 16-17 October.

EspinA, A. (1994): «Una Política de Cooperación para los Sistemas Productivos Locales», Revista de Estudios Regionales de la Comunidad de Madrid, n. ${ }^{\circ}$ 11, 149-172.

Estevan A. (1988): La Industria Electrónica en la Comunidad de Madrid, Consejería de Economía, Comunidad de Madrid.

Fernández, A. (1998): «CASA: 75 Años de Industria Aeronáutica en Madrid», Economistas, n. ${ }^{\circ} 79,276-291$.

FILIPPI, M. (1996): «Les Systèmes Localisés de Production et d'Innovation: Une Approche en Termes de Reseaux», Revue d'Économie Régionale et Urbaine, n. ${ }^{\circ}$ 4, 727-742.

FreEMAN, C. (1991): «Networks of Innovators: A Synthesis of Research Issues», Research Policy, n. ${ }^{\circ} 20,499-514$.

GAFFARD, J. L. (1990): Économie Industrielle et de l'Innovation, Dalloz, Paris.

Gray, M.; Golob, E. y Markusen, A. (1996): «Big Firms, Long Arms, Wide Shoulders: The 'Hub-and-Spoke' Industrial District in the Seattle Region», Regional Studies, vol. 30.7, 651-666.

Heraud, J. A.; HAHN, R.; GAISER, A. y Muller, E. (1995): «Reseaux d'Innovation et Tissu Industriel Régional: Une Comparaison Alsace/Bade-Wurtemberg», en: HAUDEVILLE, B.; HERAUd, J. A. y Humbert, M. (Eds.), Technologie et Performances Économiques, 97-121, Economica, Paris.

Heraud, J. A. y NANopoulos, K. (1994): «Les Reseaux de l'Innovation dans les PMI: Illustration sur le Cas de l'Alsace», Revue Internationale PME, vol. 7, nos 3 y 4 .

Hidalgo Nuchera, A. (1997): La I+D en la Industria Electrónica de Madrid. Recursos Públicos y Privados, Documento de Trabajo del IEG-CSIC, Proyecto 06/0092/1997, Madrid.

ImADE (1995): Centro de Empresas Innovadoras: Parque Tecnológico de Madrid, Comunidad de Madrid, Agencia de Desarrollo Regional, Madrid (citado par van het Kaar et Kok (1996)).

INE (1996): Estadísticas de Producción Industrial, Instituto Nacional de Estadística, Madrid.

- (1997): Encuesta de Empresas Innovadoras, Instituto Nacional de Estadística, Madrid

IRAnzo, J. E.; Río, C. Del y Molina, M. (1993): «Madrid, entre Dos Modelos de Desarrollo», Papeles de Economía Española, n. ${ }^{\circ}$ 56, 256-277.

JARILLO, J. C. (1988): «On Strategic Networks», Strategic Management Journal, vol 9, 3141.

KAAR, J. van het y KoK, R. (1996): The Technology Park of Madrid in Tres Cantos, Document mimeo., Economische Geografie \& Regionale Economie, Vakgroep Strategisch Management \& Markttheorie, Universiteit van Amsterdam. 
LoREnzonI, G. (1982): «From Vertical Integration to Vertical Desintegration», Paper presentado a Strategic Management Society Conference, Montreal (citado par Jarillo (1988)).

MARTínez ROMERO, R. (1998): «Dinámica del Sector de Fabricación de Material Electrónico en la Comunidad de Madrid, Una Aproximación Metodológica», en El Futuro de la Industria en la Comunidad de Madrid, Instituto de Estadística, Consejería de Hacienda de la CAM, Madrid.

Mella-Márquez, J. M. y Állvarez-GonZÁlez, M. I. (1998): «Parques Tecnológicos y Política Industrial en Madrid», en El Futuro de la Industria en la Comunidad de Madrid, Instituto de Estadística, Consejería de Hacienda de la CAM, Madrid.

MoNATERI, J. C. y RUFFIEUX, B. (1996): «Le Temps de la Quasi-Intégration: Une Appoche Dynamique», en: Jacques-Laurent Ravix (Coord.) Coopération entre les Entreprises et Organisation Industrielle, 77-110, CNRS Éditions, París.

MuRILlo CASTILLEJO, J. (1997): «Factores de Localización en la Industria Electrónica de la Región Madrileña», Estudios Geográficos, Tomo LVII, n. 226, 77-93, enero-marzo 1997,

ONDATEGUI RUBIO, J. C. (1998): «La Nueva Industria en Tres Cantos», in El Futuro de la Industria en la Comunidad de Madrid, Instituto de Estadística, Consejería de Hacienda de la CAM, Madrid.

RAMA, R. (1992): «La Industria de Equipos de Telecomunicación en Madrid», Alfoz, $\mathbf{n}^{\circ} 89$.

- (1998), Estrategias de las empresas electrónicas en los años noventa. Situación en los mercados internacionales y españoles, Documento de Trabajo del IEG-CSIC, Madrid.

- (1999): Grupos y Estrategias en la Electrónica Internacional, Documento de Trabajo del IEG-CSIC, Madrid.

SANZ MENÉNDEZ, L. y ARIAS E. (1999): Concentración y Especialización Regional de las Capacidades Tecnológicas: Un análisis a través de las Patentes Europeas, Documento de Trabajo del IESA (CSIC), Madrid.

SAXONIAN, A. (1992): «Contrasting Patterns of Business Organisation in Silicon Valley», Environment and Planing $D, 10,377-391$.

SignORINI, L. F. (1994): «The Price of Prato, or Measuring the Industrial District Effect», Papers in Regional Science, 73, 4, 369-392.

STORPER, M y HARRISON, B. (1991): «Flexibility, Hierarchy and Regional Development: The Changing Structure of Industrial Production Systems and Their Forms of Governance in the 1990s», Research Policy, n. ${ }^{\circ} 20,407-422$.

SuÁREz-VILLA, L. y RAMA, R. (1996): «Outsourcing, R\&D and the Pattern of Intra-metropolitan Location: The Electronics Industries of Madrid», Urban Studies, Vol. 33, n. ${ }^{\circ}$, $1155-1197$.

RESUMEN: Este artículo determina la presencia de dos núcleos, de características diferentes en cuanto a su estructura y relaciones inter-empresariales, en la industria electrónica de la Comunidad Autónoma de Madrid: Uno de ellos, formado por establecimientos de menor tamaño se asienta principalmente en el centro y se forma por la relación estrecha mantenida por las firmas dentro de redes de subcontratación. El otro, integrado por establecimientos de mayores dimensiones, se localiza fundamentalmente en la periferia de la CAM. Los establecimientos que lo integran, aunque también recurran a la subcontratación, exhiben una mayor propensión a relacionarse con sus casas matrices y Universidades, además de presentar una mayor potencialidad de encadenamiento supraregional con otras empresas que el núcleo del centro.

ABSTRACT: This article determines the existence of two different groups of firms in the electronics industries of Madrid. The first, which is comprised of smaller establish- 
ments, is chiefly located in metropolitan areas and is formed up by company linkages in outsourcing networks. Integrated by larger establishments, the other is chiefly located in peripheral locations of the Region. The establishments that form the latter group show greater propensity to relate to their parent firms; in addition, they also display greater potential for developing linkages with supra-regional firms.

RÉsumÉ: Cet article essaye d'établir l'existence de deux noyaux distincts dans l'industrie électronique de Madrid. Formé principalement par des établissements de petite taille, le premier est surtout localisé dans la ville de Madrid et s'articule autour de réseaux de soustraitance. Intégré par des établissements de plus grande taille, le deuxième noyau est plutôt localisé dans la périphérie madrilène, en même temps que les entreprises quie le forment ont surtout rapport avec leur maison-mère et montrent davantage de propension à intégrer des réseaux supra-régionaux. 